\title{
ON STRESS-STRAIN RELATIONS FOR ISOTROPIC RIGID PERFECTLY PLASTIC SOLIDS*
}

\author{
By J. L. DAIS (University of Minnesota)
}

Abstract. Sufficient conditions under which principal directions of stress and strain rate must coincide are established rigorously. It is the coincidence of these directions which permits a proper interpretation of principal strain rate components in principal stress space.

Introduction. A rigid perfectly plastic solid is characterized by a "yield" or "limit" surface $f\left(\sigma_{i j}\right)=0$ in stress component space which encloses allowable stress states. The strain rate tensor $\dot{\epsilon}_{i j}$ must vanish if $\sigma_{i j}$ is inside the surface and, if strain rate component coordinates are superposed upon stress component coordinates, must be normal to the surface if $\sigma_{i j}$ is on the surface.

If the yield surface is that of Mises (that is, if $f\left(\sigma_{i j}^{\prime}\right)=\frac{1}{2} \sigma_{i j}^{\prime} \sigma_{i i}^{\prime}$, where $\sigma_{i j}^{\prime}$ is the stress deviator tensor) then normality requires that for some scalar $\lambda, \dot{\epsilon}_{i j}=\lambda\left(\partial f / \partial \sigma_{i i}\right)=\lambda \sigma_{i j}^{\prime}$; coincidence of principal directions of stress and strain rate is then immediate since the principal axes of the stress and stress deviator tensors coincide.

More generally, if $f$ is a function of stress invariants which is differentiable in the components of $\sigma_{i j}$ then the principal directions of $\sigma_{i j}$ and $\partial f / \partial \sigma_{i j}$ conicide; by normality, then, the axes of $\sigma_{i j}$ and $\dot{\epsilon}_{i i}$ coincide. Alternatively, a stress-strain rate equation of the form $\dot{\boldsymbol{\varepsilon}}=\mathbf{f}(\boldsymbol{\delta})$ can be postulated and the material taken to be isotropic if and only if for all orthogonal transformations $s$ the equation $s \dot{\varepsilon} s^{-1}=\lambda \mathbf{f}\left(s \boldsymbol{\delta} s^{-1}\right)$ holds, where $s^{-1}$ is the inverse of $s$. From this definition of "response symmetry", coincidence follows from a well-known theorem [1].

Hill [2] and Shield [3], among others, have taken coincidence for the rigid perfectly plastic Tresca material on the premise of material isotropy but did not supply a proof. Neither of the results quoted above are applicable since the first requires a differentiable yield function and the second a unique correspondence between the tensors of stress and strain rate or their deviators. Moreover, coincidence does not hold for an isotropic frictional material [4], which obeys the verbal hypotheses upon which coincidence for the Tresca material is generally taken.

In Sec. 1 a pictorial description of the rigid perfectly plastic Tresca material is given and it is explained why coincidence does not hold for the conceptually similar frictional material. In Sec. 2 it is shown that coincidence must hold for a rigid perfectly plastic material if the question of "whether or not a stress state represents yield" is to be independent of the relative orientation of material and principal stress directions.

1. The Tresca and frictional materials. The relation between the tensors of stress and strain rate for the Tresca material is explained in Fig. 1(a) if the principal stress components are ordered as $\sigma_{1}>\sigma_{2}>\sigma_{3}$. Deformation occurs by simple shearing in the direction of the shear stress vector $\mathrm{T}$ if the magnitude $\tau$ of $\mathrm{T}$ reaches a critical value $k$. Since the planes of maximum shearing, called slip planes, are orthogonal, the simple

*Received March 3, 1968. The conclusions presented in this paper were obtained in the course of research sponsored by the National Science Foundation under Grant GK 1013 to Brown University. 


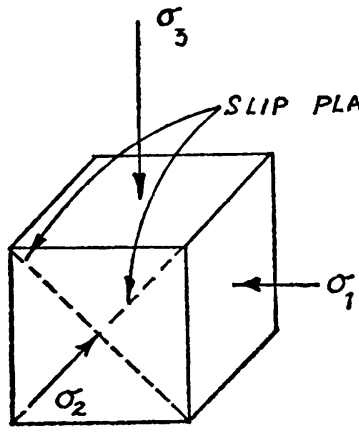

(i)

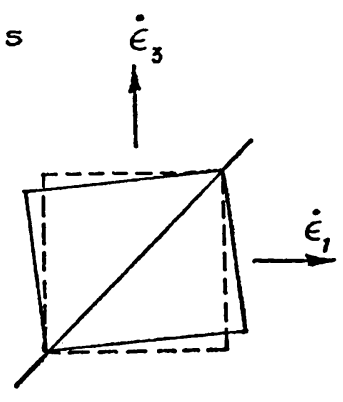

(ii)

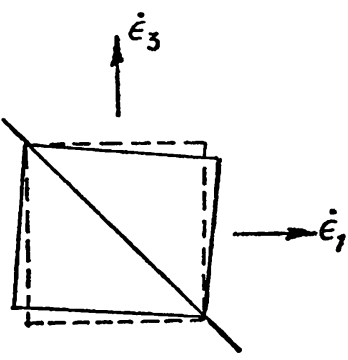

(iii)

FIG. 1(a). Rigid perfectly plastic Tresca material

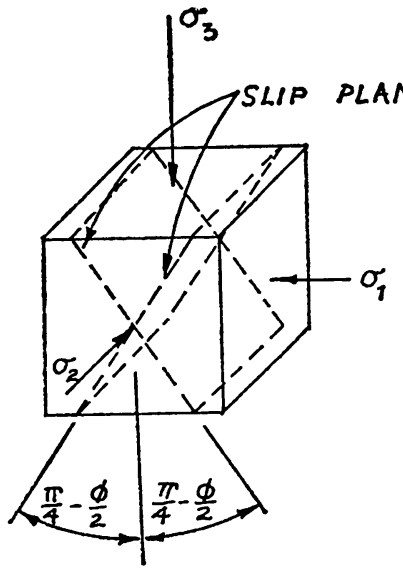

(i)

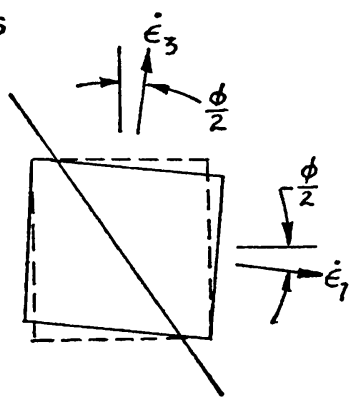

(ii)

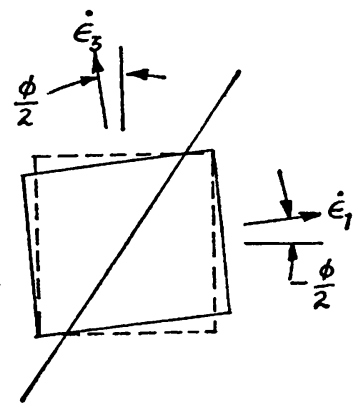

(iii)

Fig. 1(b). Frictional material; $\varphi$ :angle of internal friction

Figure 1. Configuration of a block before and after a small homogeneous deformation. Displacements are chosen so that block center remains stationary and one of the sets of zero extension does not rotate.

shearing occurs simultaneously on both sets of slip planes; the first order difference in the deformed configurations of Figs. (ii) and (iii) is in rigid rotation only.

Should two principal stresses be equal, say $\sigma_{1}=\sigma_{2}$, then $\tau$ reaches the value $k$ simultaneously on all planes with normal inclined at $\pi / 4$ to the $\sigma_{3}$ direction; deformation can occur by simple shearing along any of these mutually perpendicular sets of planes. Deformations other than plane are achieved by successive simple shears on different mutually perpendicular sets of planes. The strain rate tensor is then taken as a suitable time average of the successive simple shears. The directions of $\sigma_{3}$ and $\dot{\epsilon}_{3}$ thus remain coincident.

The relation between the tensors of stress and strain rate for plane motions of a frictional material is explained in Fig. 1(b). Deformation occurs once again by simple shearing on a slip plane but slip planes and planes of maximum shear stress do not coincide. Neither set has a material preference for shearing, so the principal axes of strain rate 
can be inclined either as in Fig. (ii) or in Fig. (iii). For this material, strain rate is not normal to the limit surface.

2. Coincidence for isotropic rigid perfectly plastic materials. No regularity assumptions on the yield surface are necessary for the following proof of coincidence. The notions of isotropy and normality will not be made precise; rather, necessary conditions for their satisfaction are stated.

A seemingly reasonable necessary condition for a perfectly plastic material to be isotropic is the following: if $\sigma_{i j}^{0}$ is an arbitrary point on a yield surface, then $\sigma_{i i}$ is on the surface also if $\sigma_{i j}$ differs from $\sigma_{i j}^{0}$ in principal directions but not principal values.

Say that at a point on a hypersurface there are one or more hypercurves on the surface with uniquely determined tangent directions. The hypersurface itself need not be regular there. A seemingly reasonable necessary condition for a vector to be "normal" to the hypersurface there is that the inner product of the vector with uniquely determined tangent directions should vanish.

Without loss of generality, take $x_{1}, x_{2}$, and $x_{3}$ axes to coincide respectively with the directions of $\sigma_{1}^{0}, \sigma_{2}^{0}$, and $\sigma_{3}^{0}$ as shown in Fig. 2. The hypercurve defined by

$$
\left\|\sigma_{i i}\right\|=\left[\begin{array}{ccc}
\sigma_{11} & \sigma_{12} & \sigma_{13} \\
\sigma_{12} & \sigma_{22} & \sigma_{23} \\
\sigma_{13} & \sigma_{23} & \sigma_{33}
\end{array}\right]=\left[\begin{array}{ccc}
\sigma_{3}^{0}+\left(\sigma_{1}^{0}-\sigma_{3}^{0}\right) \cos ^{2} \alpha & 0 & \left(\sigma_{1}^{0}-\sigma_{3}^{0}\right) \cos \alpha \sin \alpha \\
0 & \sigma_{2}^{0} & 0 \\
\left(\sigma_{1}^{0}-\sigma_{3}^{0}\right) \cos \alpha \sin \alpha & 0 & \sigma_{1}^{0}-\left(\sigma_{1}^{0}-\sigma_{3}^{0}\right) \cos ^{2} \alpha
\end{array}\right]
$$

is generated by the rotation of principal directions of Fig. 2 and thus lies on the yield

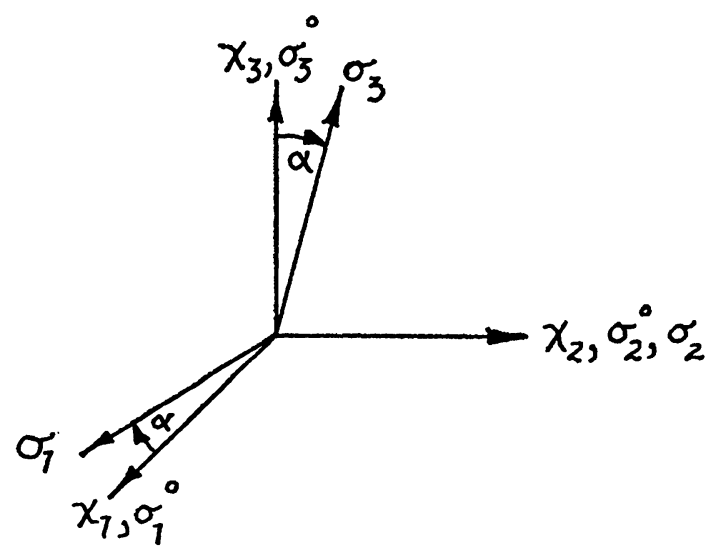

Figure 2. Principal stress directions in physical body before and after rotation about $x_{2}$ axis.

surface. The direction of the tangent vector to the hypercurve is given uniquely by $d \sigma_{i j} / d \alpha$; thus if the strain rate tensor $\dot{\epsilon}_{i j}^{0}$ at $\sigma_{i j}^{0}$ is normal to the yield surface, then $\left(d \sigma_{i j} /\right.$ $d \alpha) \dot{\epsilon}_{i j}^{0}=0$ there.

However, it follows from differentiating Eq. (1) that

$$
\left\|\frac{d \sigma_{i i}}{d \alpha}\right\|=\left[\begin{array}{ccc}
-2\left(\sigma_{1}^{0}-\sigma_{3}^{0}\right) \cos \alpha \sin \alpha & 0 & \left(\sigma_{1}^{0}-\sigma_{3}^{0}\right)\left(\cos ^{2} \alpha-\sin ^{2} \alpha\right) \\
0 & 0 & 0 \\
\left(\sigma_{1}^{0}-\sigma_{3}^{0}\right)\left(\cos ^{2} \alpha-\sin ^{2} \alpha\right) & 0 & 2\left(\sigma_{1}^{0}-\sigma_{3}^{0}\right) \cos \alpha \sin \alpha
\end{array}\right]
$$


and since $\alpha=0$ at $\sigma_{i j}=\sigma_{i j}^{0}$ that

$$
\left(d \sigma_{i j} / d \alpha\right) \dot{\epsilon}_{i j}^{0}=2\left(\sigma_{1}^{0}-\sigma_{3}^{0}\right) \dot{\epsilon}_{13}^{0} .
$$

If $\dot{\epsilon}_{13} \neq 0$, a contradiction has thus been attained provided $\sigma_{1}^{0} \neq \sigma_{3}^{0}$. Similarly, by considering hypercurves consisting of stress points $\sigma_{i j}$ which are rotated versions of $\sigma_{i i}^{0}$ about the $\sigma_{1}^{0}$ and $\sigma_{3}^{0}$ axes, the possibilities of $\dot{\epsilon}_{12} \neq 0$ and $\dot{\epsilon}_{23} \neq 0$ can be ruled out provided respectively that $\sigma_{1}^{0} \neq \sigma_{2}^{0}$ and $\sigma_{2}^{0} \neq \sigma_{3}^{0}$. Thus, as was shown pictorially for the Tresca material in the previous section, the principal axes of stress and strain rate coincide if all three principal stress components are different.

In the degenerate case of equality of two principal stress components, say $\sigma_{2}^{0}=\sigma_{3}^{0}$, then $\dot{\epsilon}_{12}^{0}=\dot{\epsilon}_{13}^{0}=0$ follows as before. Thus, the direction of $\sigma_{1}^{0}$ is also a direction of principal strain rate and the directions of $\dot{\epsilon}_{2}^{0}$ and $\dot{\epsilon}_{3}^{0}$ lie in the plane of $\sigma_{2}^{0}$ and $\sigma_{3}^{0}$. Coincidence in the sense that the directions of $\dot{\epsilon}_{2}^{0}$ and $\dot{\epsilon}_{3}^{0}$ can be arbitrarily labelled as directions of $\sigma_{2}^{0}$ and $\sigma_{3}^{0}$ is attained. Coincidence in this sense was obtained for the Tresca material by the pictorial description of the previous section.

In the coincidence proof, curves lying on a yield surface were generated by rotating two of the principal stress directions about the third; more generally, curves lying on the yield surface can be generated by arbitrary rotations of the principal stress directions. It is interesting to note that all such curves correspond to a single point of principal stress space.

For the Mises material, normality implies further that the components of the strain rate tensor are proportional to the components of the stress deviator tensor. This conclusion is of course not valid for the Tresca material.

Acknowledgments. The author acknowledges Professor D. C. Drucker for valuable discussions.

\section{REFERENCES}

[1] J. Serrin, Mathematical principles of classical fluid mechanics, Handbuch der Physik, Bd. VIII/1, Springer, Berlin-Göttingen-Heidelberg, 1959, pp. 125-263

[2] R. Hill, The mathematical theory of plasticity, Macmillan, New York, 1950

[3] R. T. Shield, On the plastic flow of metal under conditions of axial symmetry, Proc. Roy. Soc. A 233, 267-287 (1955)

[4] J. L. Dais, An isotropic frictional theory for a granular medium with or without cohesion, Brown University Tech. Report No. NSF-GK1013/6 